\title{
Change in knee structure and change in tibiofemoral joint space width: a five year longitudinal population-based study
}

Joanna Hall ${ }^{1 \dagger}$, Laura L. Laslett ${ }^{1 *}$, Johanne Martel-Pelletier ${ }^{2}$, Jean-Pierre Pelletier ${ }^{2}$, François Abram³ , Chang-Hai Ding $^{1,4,5}$, Flavia M. Cicuttini ${ }^{4}$ and Graeme Jones ${ }^{1}$

\begin{abstract}
Background: Change in knee cartilage volume is frequently used as a proxy for change in knee joint space width over time, but longitudinal data on these associations is limited. We aimed to determine whether change in knee cartilage volume, new or worsening meniscal extrusion (ME), meniscal tears and cartilage defects over 2.4 years correlated with change in joint space width (JSW) over 5 years in older community dwelling adults.

Methods: Participants $(n=153)$ had their right knee imaged using MR imaging and $x$-ray at baseline, and after 2.4 years (MRI) and 5 years ( $\mathrm{x}$-ray). Cartilage volume, cartilage defects, meniscal extrusions and meniscal tears were assessed on sagittal T1-weighted fat-suppressed MRI. JSW was assessed using standard fixed semi-flexed view radiographs, and scored on those with adequate alignment.

Results: Participants were 51-79 (mean 62) years old; $48 \%$ were female. Cartilage volume reduced over time (medial $-134 \pm 202 \mu \mathrm{L} /$ year, lateral $-106 \pm 165 \mu \mathrm{L} /$ year, $p<0.001$ ), as did JSW (medial $-0.05 \pm 0.16 \mathrm{~mm} /$ year, lateral $-0.12 \pm 0.24 \mathrm{~mm} /$ year, $p<0.001)$. In multivariable analysis, the only consistent predictor of change in JSW was new or worsening ME (medial tibia $\mathrm{R}^{2} 3.1 \%, p=0.031$; medial femur $\mathrm{R}^{2} 3.2 \%, p=0.024$ ); change in cartilage volume correlated with change in JSW laterally $\left(R^{2} 4.8 \%, p=0.007\right)$ and was borderline medially ( $\left.R^{2} 2.2 \%, p=0.064\right)$; there was no association for meniscal tears or cartilage defects. The magnitude of these associations were similar albeit somewhat greater for ME in participants with radiographic OA $\left(R^{2} 6.2 \%, p=0.017\right)$.

Conclusion: Change in ME and cartilage volume weakly predict change in JSW, but the vast majority of the variation remains unexplained. Since MRI examines cartilage directly while radiographs examine it indirectly, these results cast doubt on the validity of using JSW as a proxy measure of cartilage loss.
\end{abstract}

Keywords: Cartilage, Meniscus, Extrusion, X-ray, MRI

\section{Background}

Osteoarthritis (OA) is a major cause of pain and functional limitations and disability worldwide [1]. Diagnosis is based on a combination of symptoms, clinical signs and radiographic abnormalities [2]. Change in joint space width (JSW) at the tibiofemoral joint has historically been considered a good measure of change in cartilage volume. It is currently the gold standard for assessing osteoarthritis

\footnotetext{
* Correspondence: Laura.Laslett@utas.edu.au

${ }^{\dagger}$ Equal contributors

${ }^{1}$ Menzies Institute for Medical Research, University of Tasmania, Private Bag

23, Hobart, Tasmania 7000, Australia

Full list of author information is available at the end of the article
}

disease modification in clinical trials [3], and is mandated by the Food and Drug Administration and European Medications Agency as a proxy endpoint to determine efficacy of disease modifying osteoarthritis drugs. In crosssectional studies, cartilage volume assessed by magnetic resonance imaging (MRI) and JSW as assessed by radiograph are strongly correlated. However, JSW is also associated with meniscal pathology [4-7], and cartilage defects [8], suggesting that multiple abnormalities contribute to narrowing of joint space width (JSW) over time. The presence of radiographic $\mathrm{OA}$ also predicts patients who lose cartilage faster; this has implications for participant selection in clinical trials $[9,10]$. 
A key remaining question is whether radiographic JSW is the most appropriate measure for assessing change in knee OA over time. Some studies have shown significant structural change at the tibiofemoral joint over time, such as trials involving glucosamine, doxycycline and chondroitin [11-14]. However, in a trial investigating the effect of risedronate on OA progression, there was minimal change in the placebo arm over 2 years, despite large numbers of patients and state of the art protocols [15]. MRI-based cartilage loss has greater sensitivity to change [16-18]; than change in radiographic JSW [19]. However, these assessments come from different cohorts; there is limited longitudinal data comparing change in radiographs with change in MRI within cohorts. Two studies reported weak but statistically significant correlation between changes in JSW and cartilage volume at 1 year in OA cohorts, using the fixed-flexion radiographic techniques [17, 20]. Both Cicuttini and Raynauld reported no correlation between $\mathrm{x}$-ray change and cartilage volume change on MRI over 2 years, using different methodologies: standing protocol radiographs [21] and a fluoroscopically guided AP semiflexed protocol [22]. Hunter et al [23] assessed predictive value of cartilage score on change in medial JSN in patients with symptomatic knee OA over 30 months, using fluoroscopic positioning and fixed flexion. They found that cartilage score contributed to change in JSN beyond age sex and BMI, but that most of the variability in JSN remained unexplained [23]. Since MRI has been directly validated for cartilage volume measurement, this raises the possibility that JSW is not a sufficiently adequate measure of cartilage to qualify it for use as an outcome measure in clinical trials.

Moreover, data in the literature also reported that meniscal position and cartilage morphology score also contribute to variance in JSW $[7,23]$. However, to our knowledge there have been no prospective studies which have determined the quantitative contribution of meniscal extrusion to narrowing of JSW over a period as long as 5 years. Therefore, this study aimed to determine whether change in cartilage volume and other structural factors, including new or worsening meniscal extrusion (ME), worsening meniscal tears, and worsening cartilage defects over 2.4 years predicted change in JSW over 5 years in participants from a randomly selected cohort of community dwelling older adults with and without radiographic OA.

\section{Methods}

\section{Study design and setting}

The Tasmanian Older Adult Cohort (TASOAC) study is an ongoing prospective population-based study in southern Tasmania, Australia, which began in 2002.

\section{Study participants}

Men and women aged 50-80 years were randomly selected from the roll of electors in southern Tasmania (population 229,000), a comprehensive population listing, using sex-stratified simple random sampling without replacement (response rate $57 \%$ ). Permission to access the roll of electors was granted by the Australian Electoral Commission. Persons were excluded if they were institutionalized, or had contraindications to MRI. The study was approved by the Southern Tasmanian Health and Medical Human Research Ethics Committee, and written informed consent was obtained from all participants. Baseline measurements (Phase 1) were conducted from April 2002 to September 2004. Follow up data (Phase 2 and 3) was collected 2.4 (range 1.7 to 2.9) and 5 years (range 4.6 to 5.9) later.

The current study consists of a sample of 153 participants who had data for $\mathrm{x}$-rays and MRI at baseline and follow-up with adequately aligned radiographs (see Table 1).

\section{Outcome measures \\ Knee radiographs}

Standing anteroposterior semiflexed right knee radiographs with $10-15^{\circ}$ of fixed knee flexion were performed at baseline and after 5 years. Radiographs were viewed on Osiris software (University Hospital of Geneva, Switzerland) and scored paired by one investigator $(\mathrm{JH}$, after instruction from GJ), blinded to MRI data but not to chronological order. Measurements of the minimum JSW (mJSW) at the lateral and medial compartment were performed to the nearest $0.1 \mathrm{~mm}$, using $200 \%$ magnification and a digital calliper in the image processing package, and enhancement to improve cortical demarcation. mJSW was determined as the narrowest, non-osteophytic space of the lateral and medial compartments of the right knee using a modified version of the method of Lequesne [24], and using bony margins as described by Buckland-Wright. [25] Briefly, the femoral boundaries were the distal convex margin of the femoral condyles, and the tibial boundaries the bright radiodense band of the subchondral cortex of the tibia. The reader selected a minimum of five points in each compartment, with the smallest reading used. This technique is similar to the "calculated minimum" technique, which is more accurate than measurement of the narrowest site using visual assessment alone [26]. Intraclass correlation (ICC) was excellent, ranging from 0.92-0.99 in a random sample of 20 participants separated in time.

In the absence of fluoroscopic guidance, radiographs were limited to those which were well aligned (67 \% of those with films at both time points) [27], defined as tibial inter-rim distance of the medial tibial plateau 
Table 1 Participant characteristics

\begin{tabular}{|c|c|c|}
\hline & & $\begin{array}{l}\text { Mean (SD) } \\
n=153\end{array}$ \\
\hline \multicolumn{2}{|l|}{ Gender } & $48 \%$ female \\
\hline \multicolumn{2}{|c|}{ Age (years) } & $62.2(7.0)$ \\
\hline \multicolumn{2}{|c|}{ BMI $\left(\mathrm{kg} / \mathrm{m}^{2}\right)$} & $27.8(4.7)$ \\
\hline \multicolumn{2}{|c|}{ Any radiographic OA (\%) } & 56.0 \\
\hline \multicolumn{2}{|c|}{ Osteophyte, any site (\%) } & 9.2 \\
\hline \multicolumn{2}{|c|}{ Medial JSW (mm) } & $4.73(0.97)$ \\
\hline \multicolumn{2}{|c|}{ Lateral JSW (mm) } & $6.76(1.37)$ \\
\hline \multicolumn{3}{|c|}{ JSN scores (OARSI grade) (\%) } \\
\hline \multirow[t]{4}{*}{ Medial } & 0 & 49.7 \\
\hline & 1 & 41.2 \\
\hline & 2 & 8.5 \\
\hline & 3 & 0.7 \\
\hline \multirow[t]{4}{*}{ Lateral } & 0 & 83.7 \\
\hline & 1 & 13.1 \\
\hline & 2 & 2.0 \\
\hline & 3 & 1.3 \\
\hline \multicolumn{3}{|c|}{ Annual change in joint space width: } \\
\hline \multicolumn{2}{|c|}{ Medial (mm) } & $-0.05(0.16)$ \\
\hline \multicolumn{2}{|c|}{ Medial (\%) } & $-1.2(3.9)$ \\
\hline \multicolumn{2}{|c|}{ Lateral $(\mathrm{mm})$} & $-0.12(0.24)$ \\
\hline \multicolumn{2}{|c|}{ Lateral (\%) } & $-1.8(3.9)$ \\
\hline \multicolumn{2}{|c|}{ Medial cartilage volume (tibia + femur, $\mu \mathrm{L}$ ) } & $6296(1523)$ \\
\hline \multicolumn{2}{|c|}{ Lateral cartilage volume (tibia + femur, $\mu \mathrm{L}$ ) } & $7080(1671)$ \\
\hline \multicolumn{3}{|c|}{ Annual change in cartilage volume: } \\
\hline \multicolumn{2}{|c|}{ Medial $(\mu \mathrm{L})$} & $-134(202)$ \\
\hline \multicolumn{2}{|c|}{ Medial (\%) } & $-2.1(3.0)$ \\
\hline \multicolumn{2}{|c|}{ Lateral $(\mu \mathrm{L})$} & $-106(165)$ \\
\hline \multicolumn{2}{|c|}{ Lateral (\%) } & $-1.6(2.5)$ \\
\hline \multicolumn{3}{|c|}{ Meniscal extrusions } \\
\hline \multicolumn{2}{|c|}{ Medial - present at baseline (\%) } & 16.3 \\
\hline \multicolumn{2}{|c|}{ Medial - worsening extrusion (\%) } & 9.2 \\
\hline \multicolumn{2}{|c|}{ Lateral - present at baseline (\%) } & 2.0 \\
\hline Later & n (\%) & 0.7 \\
\hline Menisca & & \\
\hline Medi & (\%) & 98.0 \\
\hline Medi & & 10.5 \\
\hline Later & (\%) & 96.7 \\
\hline Later & & 15.0 \\
\hline Cartilag & & \\
\hline Medi & (\%) & 23.5 \\
\hline Medi & & 37.7 \\
\hline Later & (\%) & 21.6 \\
\hline Later & & 36.4 \\
\hline
\end{tabular}

Table 1 Participant characteristics (Continued)

\begin{tabular}{ll}
\hline Cartilage thickness (average, tibia \& femur) & $(n=67)$ \\
Medial (mm) & $3.07(0.41)$ \\
Lateral (mm) & $3.33(0.41)$ \\
Annual change in cartilage thickness & \\
Medial (mm) & $-0.17(0.27)$ \\
Medial (\%) & $-6.04(8.45)$ \\
Lateral (mm) & $-0.26(0.3)$ \\
Lateral $(\%)$ & $-8.12(8.69)$ \\
\hline
\end{tabular}

Change in cartilage and meniscal extrusion is over a time period of 2.4 years Change in joint space width is over a time period of 5 years Right knees only

varying by $\leq 2 \mathrm{~mm}$ between films, measured at the midpoint of the medial compartment.

$\mathrm{X}$-rays were scored individually for osteophytes and narrowing of JSW on a scale of $0-3(0=$ normal, $3=$ severe $)$ according to the Osteoarthritis Research Society International (OARSI) atlas [28] as previously described [29]. Intraobserver repeatability was acceptable (ICCs of 0.65 to 0.85 in 40 participants) [29]. The presence of radiographic $\mathrm{OA}$ was defined as any score of $\geq 1$.

\section{Magnetic resonance imaging}

Magnetic resonance imaging (MRI) of the right knee was acquired using a 1.5 Tesla whole-body MRI unit (Picker, Cleveland, OH, USA) using a commercial transmit-receive extremity coil.

Tibial cartilage volume was assessed at baseline and follow up by a trained observer on Osiris software as previously described [30, 31] using T1-weighted fat suppressed 3-dimensional gradient recall acquisition in the steady state, flip angle $55^{\circ}$, repetition time $58 \mathrm{msec}$, echo time $12 \mathrm{msec}$, field of view $16 \mathrm{~cm}, 60$ partitions, $512 \times 512-$ pixel matrix, acquisition time $11 \mathrm{~min} 56 \mathrm{~s}$, and 1 acquisition. Sagittal images were obtained at a partition thickness of $1.5 \mathrm{~mm}$ and in-plane resolution of $0.31 \times 0.31 \mathrm{~mm}(512 \times 512$ pixels $)$. The volumes of individual cartilage plates (medial tibia and lateral tibia) were isolated from the total volume by manually drawing disarticulation contours around the cartilage boundaries on a section by section basis (see Fig. 1 for an image of representative segmentation). These data were then resampled by means of bilinear and cubic interpolation (area of $312 \times 312 \mathrm{~mm}$ and $1.5 \mathrm{~mm}$ thickness, continuous sections) for the final 3-D rendering. The coefficient of variation $(\mathrm{CV})$ was $2.1 \%$ for the medial tibia and $2.2 \%$ for the lateral tibia [30].

Femoral cartilage volume was determined by means of image processing on an independent workstation using Cartiscope ${ }^{\mathrm{TM}}$ (ArthroVision Inc., Montreal, Quebec, Canada), as previously described [4, 32, 33]. The segmentation of the cartilage-synovial interfaces was carried out 


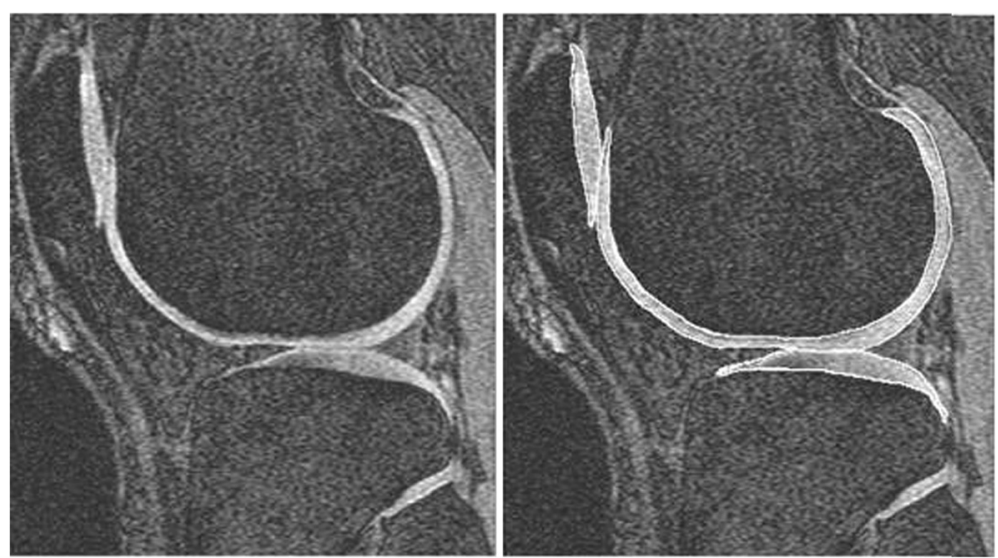

Fig. 1 A representative MR image of cartilage segmentation. The volumes of individual cartilage plates (medial tibia and lateral tibia) were isolated from the total volume by manually drawing disarticulation contours around the cartilage boundaries on a section by section basis, before being re-sampled for 3-D rendering

using a semi-automatic method under reader supervision, and with corrections when needed. Cartilage volume was evaluated directly from a standardized view of 3D cartilage geometry as the sum of elementary volumes. The CV was approximately $2 \%$ [32]. The cartilage volume assessment was done for the medial and lateral condyles delineated by the Blumensaat's line [33].

Meniscal extrusion and tears were assessed using the T1-weighted fat suppressed sequences described earlier [4]. Anterior and posterior horns of the menisci were scored using the sagittal views and the body of the menisci using reconstructed coronal views. The proportion of the menisci affected by extrusion was measured separately on the medial and lateral edges of the tibiofemoral joint space using a semi-quantitative scale. The extent of meniscal extrusion, excluding osteophytes, was evaluated for the anterior, middle, and posterior horns of the menisci in which $0=$ no extrusion, $1=$ partial extrusion and 2 =complete extrusion with no contact with the joint space (severe) [4]. The extent of meniscal tears were assessed using the following scale: $0=$ no damage; $1=$ one of three meniscal areas involved (anterior, middle, and posterior horns); 2 = two of three areas involved; $3=$ all three areas involved [4]. Change in meniscal tears were classified as improvement or no change if scores were unchanged or improved at either tibia or femur, or classified as worsening if scores increased. Reliability was excellent; intra- and inter-observer correlation coefficients ranged from $0.86-0.96$ [22].

Mean cartilage thickness for each of four regions (femur facing the medial tibia, femur facing the lateral tibia, medial tibia and lateral tibia) was assessed using custom semi-automated segmentation software, and was calculated as the mean distance from inner to outer surface. This was done from a sample of uniformly spaced points over the entire cartilage-covered surface.
Reliability was excellent: intra-observer reproducibility for mean intensity in each region was less than $1.5 \%$, with a coefficient of variation $<2.9 \%$ [34].

Cartilage defects were assessed by a trained observer at the medial tibial, medial femoral, lateral tibial, and lateral femoral sites, using the T1-weighted fat suppressed sequences described earlier, and as previously described [8]: grade $0=$ normal cartilage; grade $1=$ focal blistering and intracartilaginous low-signal intensity area with an intact surface and base; grade $2=$ irregularities on the surface or base and loss of thickness $<50 \%$; grade $3=$ deep ulceration with loss of thickness $>50 \%$; and grade $4=$ fullthickness chondral wear with exposure of subchondral bone. ICCs ranged from 0.89-0.94 for intra-observer reliability. These were dichotomised into none (grades 0 and 1 (normal/focal blistering)) or any defects (grades 2 and above). Change in cartilage defects were classified as improvement or no change if scores were unchanged or improved on the $0-4$ scale at either tibia or femur, or classified as worsening if scores increased.

\section{Statistical analysis}

We used Stata 12.1 (Stata Corp LP) for statistical analyses. Statistical significance was set as a $p$ value of $\leq 0.05$ (two tailed). Linear regression was used to assess the association between change in cartilage volume, meniscal extrusions, meniscal tears over 2.4 years and change in JSW over 5 years. $R^{2}$ values for univariable models are the $\mathrm{R}^{2}$ statistic from the linear regression. $\mathrm{R}^{2}$ values for multivariable models are the squared semipartial correlations for each individual predictor in the multivariable model, which represent the proportion of variance in the outcome that is explained by the individual predictor only. Total $R^{2}$ is the sum of the squared semipartial correlations for individual predictors, and is given by 
the adjusted $\mathrm{R}^{2}$ statistic (adjusted for degrees of freedom) in the multivariable linear regression.

\section{Results}

A total of 153 participants ( $48 \%$ female, mean age 62 years, [range 51-79]) had adequately aligned radiographs (206/307 pairs), complete MR imaging, and radiograph data of the knee at baseline and follow up. Baseline characteristics of this sample $(n=153)$ were similar to the overall TASOAC population $(n=1099)$ (age 62.2 vs 63.2 years, $p=0.14$; sex $48 \%$ female vs $49 \%$ female, $p=0.75$; BMI 27.8 vs $27.9, p=0.84$ ) (Table 1 ). Half of the participants $(56 \%, n=84)$ had at least grade 1 radiographic OA at baseline, defined as either JSN or presence of osteophytes using the OARSI atlas [28]. Medial and lateral cartilage volume significantly reduced over time (medial $-134.3 \mu \mathrm{L} /$ year $(95 \% \mathrm{CI}-166.6$ to -102.0 ), lateral $-106.2 \mu \mathrm{L} /$ year, $(95 \% \mathrm{CI}-132.5$ to -79.9$)$, as was JSW (medial $-0.05 \mathrm{~mm} /$ year (95\% CI -0.08 to -0.03 ), lateral $-0.12 \mathrm{~mm} /$ year, $(95 \% \mathrm{CI}-0.16$ to -0.08$)$. Although the majority of participants demonstrated a decrease in cartilage volume over time, JSW both decreased and (Fig. 2). In the medial compartment, $67 \%$ of participants had a decrease in JSW, $5 \%$ stayed the same, and $28 \%$ increased. Similar values were found for the lateral compartment (73\% had a decrease in JSW, $2.6 \%$ stayed the same, and $24 \%$ increased).

Change in cartilage volume over 2.4 years was a significant predictor of change in JSW over 5 years in univariate analyses $\left(\mathrm{R}^{2}=4.4 \%, p=0.009\right.$ medially; $\mathrm{R}^{2}=$ $4.9 \%, p=0.006$ laterally) (Table 2, Fig. 2a and b). However, in multivariable analysis, change in cartilage volume was a significant predictor of change in JSW only in the lateral compartment $\left(\mathrm{R}^{2} 4.8 \%, p=0.007\right.$ laterally; $\mathrm{R}^{2} 2.2 \%, p=0.064$ medially) (Table 2 ).

New or worsening meniscal extrusions (ME) occurred in 14 cases $(9.2 \%)$ medially and 1 case $(0.66 \%)$ laterally. Only the medial data was used in the analysis due to the small number of lateral ME. Of these meniscal extrusions, 9 were none to partial, 3 partial to complete and 2 none to complete extrusion. In unadjusted analyses, change in medial JSW was greater in individuals with new or worsening meniscal extrusions (Table 2, Fig. 3). In multivariable analyses, new or worsening meniscal extrusions over 2.4 years predicted change in JSW over 5 years at the medial tibial $\left(\mathrm{R}^{2} 3.1 \%, p=0.031\right)$ and medial femur $\left(\mathrm{R}^{2} 3.2 \%, p=0.024\right)$ sites, and was strongest in participants with radiographic OA (OARSI grade 21) $\left(R^{2}=6.2 \%, p=0.017\right)$, but did not reach statistical significance in the whole population at both sites $\left(\mathrm{R}^{2}\right.$ $2.4 \%, p=0.053)$, after adjustment for change in meniscal tears and cartilage defects. When predictive validity of both change in cartilage volume and ME for JSW was
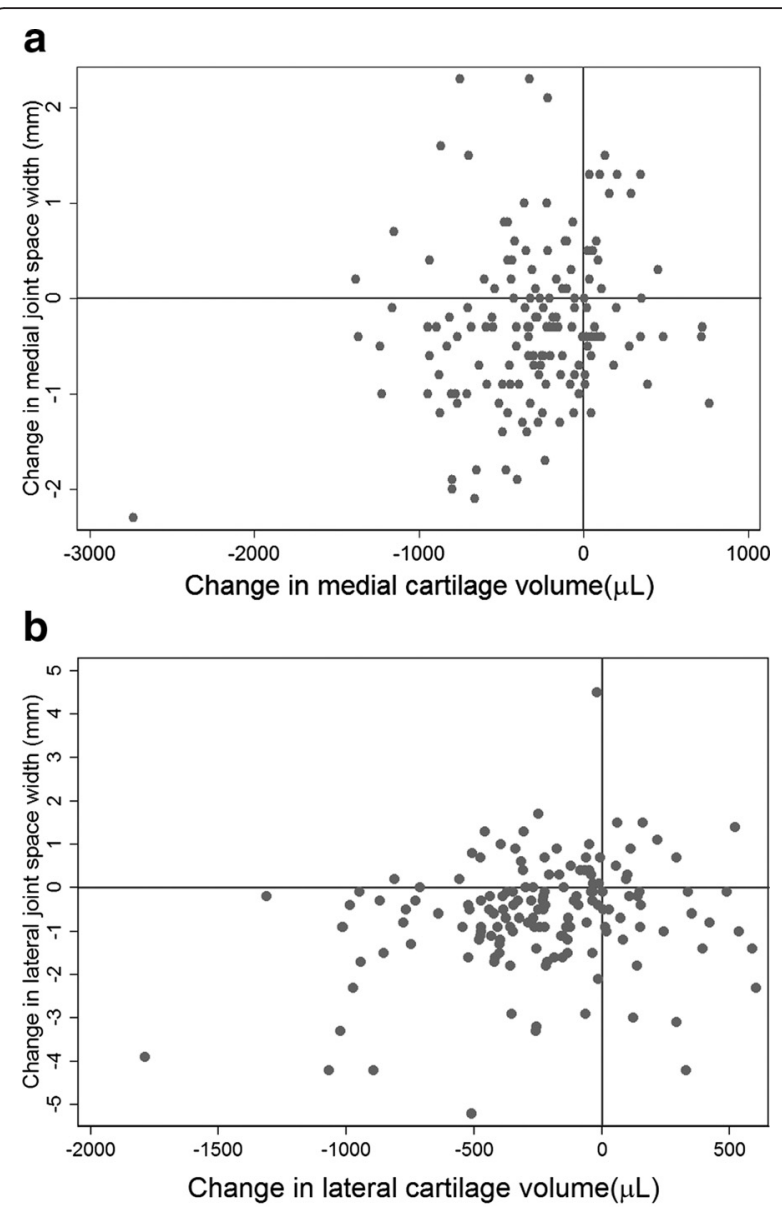

Fig. 2 Scatterplots showing the association between change in joint space width (JSW) and change in cartilage volume ( $n=153$ medial, $n=152$ lateral). In both compartments, cartilage volume decreased in 117 participants each of $a$ and b, but in 29 in a and 26 in b JSW increased despite a decrease in cartilage volume. a Medial compartment: there is a significant negative correlation, with $R^{2}=4.4 \%, p=0.009$ (unadjusted). $\mathbf{b}$ Lateral compartment: there is a significant negative correlation, with $\mathrm{R}^{2}=4.9 \%, p=0.006$ (unadjusted)

considered, they were additive (ie combined $\mathrm{R}^{2}=8.3 \%$ in those with radiographic OA).

The addition of cartilage defects or meniscal tears to the model did not reach statistical significance medially or laterally, or within any subgroup.

We repeated analyses using another marker of cartilage assessed in a subset of this cohort: cartilage thickness (Table 3). This data suggests that associations between change in cartilage thickness and change in JSW are of similar or lesser magnitude to change in cartilage volume loss and change in JSW. Similarly, total $\mathrm{R}^{2}$ in models assessing change in cartilage thickness (Table 3) is similar to, or less than values for models assessing change in cartilage volume (Table 2). Data on study participants with ROA are not shown as the number of included participants is too small $(n=40)$. 


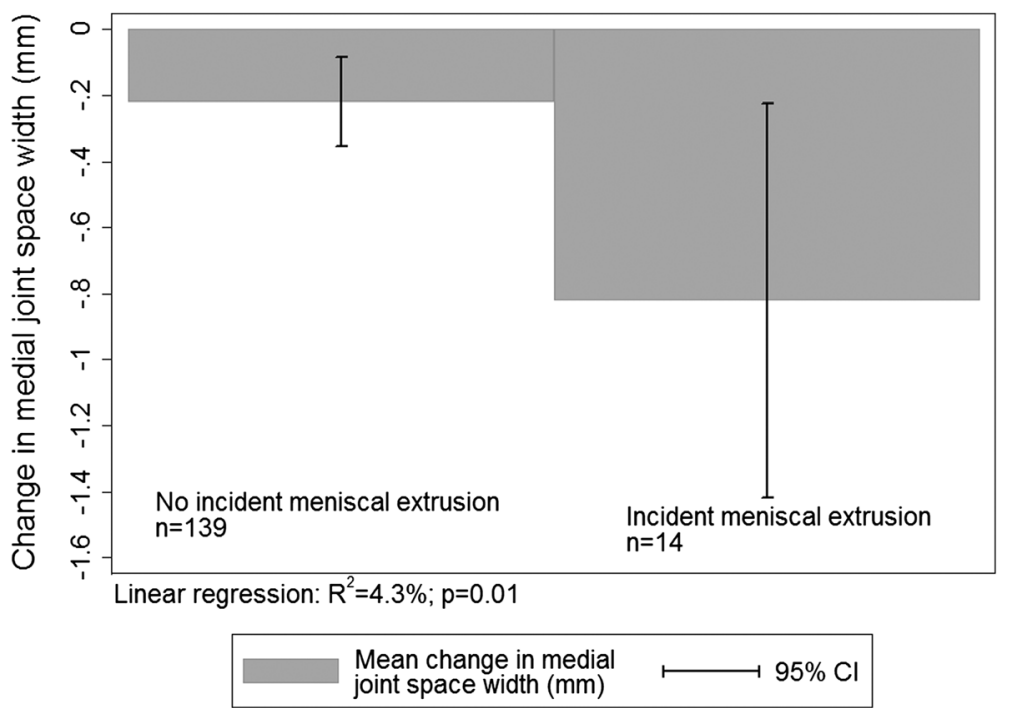

Fig. 3 Mean change in joint space width stratified by meniscal extrusion. In participants with incident meniscal extrusion at 2.4 years there was a significantly greater decrease in JSW over 5 years (unadjusted)

\section{Sensitivity analyses}

$\mathrm{X}$-ray is weight bearing, but MRI is not; therefore we further adjusted for BMI. This increased $R^{2}$ values for change in medial joint space width by small amounts: $3.0 \%$ vs $2.2 \%$ for total cartilage, and $3.0 \%$ vs $2.4 \%$ for meniscal extrusion; and $3.8 \%$ vs $2.1 \%$ for total cartilage, and $6.8 \%$ vs $6.2 \%$ for meniscal extrusion in people with radiographic OA. $\mathrm{R}^{2}$ for change in cartilage volume, meniscal extrusion and BMI were 8.3 and $17.1 \%$ for these models.

JSN in one compartment might affect change in another; therefore we assessed change in medial JSN in participants without lateral JSN at baseline $(n=126)$. Total $\mathrm{R}^{2}$ values increased by a small amount $(7.7 \%$ vs $5.1 \%)$, but $\mathrm{R}^{2}$ values for total cartilage volume were similar $\left(R^{2}=1.8 \%\right.$ vs $\left.2.2 \%\right) . R^{2}$ values for meniscal extrusion increased in magnitude $\left(\mathrm{R}^{2}=4.4, p=0.02\right.$ vs $\left.\mathrm{R}^{2}=2.4 \%, p=0.053\right)$, reaching statistical significance.

\section{Discussion}

In this 5 year longitudinal study of a population based cohort, both JSW and cartilage volume decreased significantly over time. Associations between cartilage volume loss and change in JSW at both medial and lateral compartments were weak and did not reach statistical significance at all sites. Associations were consistent in magnitude, explaining 2-13\% of the variance regardless of measurement site or stratified analysis; although not all of these attained statistical significance. The strength of the associations between ME and change in JSW was similar to the associations between change in cartilage volume and change in JSW, and was strongest in those with radiographic OA. Overall, over $80 \%$ of the variation in JSW change amongst individual study participants remains unexplained, possibly due to measurement error.

Whilst we observed an association between change in JSW and cartilage volume loss, the small magnitude of the association suggests that change in JSW over 5 years provides only a very limited reflection of change in cartilage volume over 2.4 years. Unlike radiographs, MRI allows direct visualisation of the cartilage along with other soft tissue structures, and in 3D compared to the $2 \mathrm{D}$ for radiographs. MR imaging has been validated in cadaveric studies [35], and has demonstrated a direct link with clinical outcomes such as joint replacement [36].

New or worsening cases of meniscal extrusion were uncommon in our cohort, as previously reported [7]. However, ME contributed a similar or larger amount to change in JSW as cartilage volume change at the medial compartment. Predictive validity of both cartilage volume change and ME for change in JSW was additive, but not entirely independent. In participants with radiographic $\mathrm{OA}, \mathrm{ME}$ was a stronger predictor than change in cartilage volume for change in JSW at the medial compartment. These changes were observed in relatively small numbers of participants, with only nine participants (5.8\%) having radiographic OA and ME. However, this data is consistent with studies using categorical measures of cartilage and including larger numbers of participants with OA [7].

Adding change in meniscal tears to the multivariable model added minimal additional explanatory power, and did not reach statistical significance at any site or within any subgroup. The proportion of variance explained by cartilage defects were similar to that explained by change in cartilage volume in some subgroups, but the 
Table 2 Association between change in cartilage volume, and new or worsening meniscal extrusions, meniscal tears, and cartilage defects over 2.4 years; and change in joint space width over 5 years

\begin{tabular}{|c|c|c|c|c|c|}
\hline \multirow[t]{2}{*}{ Population } & \multirow[t]{2}{*}{ Change in.. } & \multicolumn{2}{|l|}{ Medial joint space width } & \multicolumn{2}{|c|}{ Lateral joint space width } \\
\hline & & Univariable & Multivariable $^{a}$ & Univariable & Multivariable $e^{a}$ \\
\hline All participants & Tibial + femoral cartilage volume & $\mathrm{R}^{2} 4.4 \%, p=0.009$ & $\mathrm{R}^{2} 2.2 \%, p=0.064$ & $\mathrm{R}^{2} 4.9 \%, p=0.006$ & $\mathrm{R}^{2} 4.8 \%, p=0.007$ \\
\hline$(n=151$ medially, & Meniscal extrusion & $\mathrm{R}^{2} 4.3 \%, p=0.01$ & $R^{2} 2.4 \%, p=0.053$ & NA & NA \\
\hline \multirow[t]{3}{*}{$n=150$ laterally) } & Meniscal tears & $\mathrm{R}^{2} 0.19 \%, p=0.60$ & $\mathrm{R}^{2} 0.0 \%, p=0.97$ & $\mathrm{R}^{2} 1.4 \%, p=0.15$ & $\mathrm{R}^{2} 1.2 \%, p=0.18$ \\
\hline & Cartilage defects (tibia + femur) & $\mathrm{R}^{2} 1.4 \%, p=0.15$ & $\mathrm{R}^{2} 0.7 \%, p=0.29$ & $\mathrm{R}^{2} 0.26 \%, p=0.54$ & $\mathrm{R}^{2} 0.01 \%, p=0.90$ \\
\hline & & & Total R² $7.7 \%$ & & Total R $6.2 \%$ \\
\hline \multicolumn{6}{|c|}{ Subgroup: cartilage measurement site } \\
\hline Tibia & Tibial cartilage volume & $\mathrm{R}^{2} 1.7 \%, p=0.11$ & $\mathrm{R}^{2} 0.8 \%, p=0.26$ & $\mathrm{R}^{2} 3.34 \%, p=0.024$ & $\mathrm{R}^{2} 3.8 \%, p=0.017$ \\
\hline \multirow[t]{4}{*}{$(n=151)$} & Meniscal extrusion & $\mathrm{R}^{2} 4.3 \%, p=0.01$ & $\mathrm{R}^{2} 3.1 \%, p=0.031$ & NA & NA \\
\hline & Meniscal tears & $\mathrm{R}^{2} 0.19 \%, p=0.60$ & $R^{2} 0.01 \%, p=0.91$ & $R^{2} 1.35 \%, p=0.15$ & $\mathrm{R}^{2} 1.3 \%, p=0.16$ \\
\hline & Tibial cartilage defects & $\mathrm{R}^{2} 0.23 \%, p=0.56$ & $\mathrm{R}^{2} 0.02 \%, p=0.85$ & $\mathrm{R}^{2} 0.03 \%, p=0.83$ & $\mathrm{R}^{2} 0.3 \%, p=0.52$ \\
\hline & & & Total R² $5.1 \%$ & & Total R² $5.1 \%$ \\
\hline Femur & Femoral cartilage volume & $\mathrm{R}^{2} 3.8 \%, p=0.016$ & $\mathrm{R}^{2} 1.8 \%, p=0.095$ & $\mathrm{R}^{2} 1.72 \%, p=0.11$ & $\mathrm{R}^{2} 1.5 \%, p=0.13$ \\
\hline \multirow[t]{4}{*}{$(n=150)$} & Meniscal extrusion & $\mathrm{R}^{2} 4.3 \%, p=0.01$ & $\mathrm{R}^{2} 3.2 \%, p=0.024$ & NA & NA \\
\hline & Meniscal tears & $\mathrm{R}^{2} 0.19 \%, p=0.6$ & $\mathrm{R}^{2} 0.04 \%, p=0.80$ & $\mathrm{R}^{2} 1.35 \%, p=0.15$ & $\mathrm{R}^{2} 1.2 \%, p=0.19$ \\
\hline & Femoral cartilage defects & $\mathrm{R}^{2} 3.0 \%, p=0.035$ & $\mathrm{R}^{2} 2.2 \%, p=0.063$ & $\mathrm{R}^{2} 2.26 \%, p=0.07$ & $\mathrm{R}^{2} 2.2 \%, p=0.07$ \\
\hline & & & Total R $9.0 \%$ & & Total R $4.9 \%$ \\
\hline \multirow{5}{*}{$\begin{array}{l}\text { Subgroup:Radiographic } \\
\text { OA of OARSI grade } \geq 1 \\
(n=85)\end{array}$} & Tibial + femoral cartilage volume & $\mathrm{R}^{2} 7.8 \%, p=0.01$ & $\mathrm{R}^{2} 2.1 \%, p=0.16$ & $R^{2} 3.0 \%, p=0.11$ & $R^{2} 3.3 \%, p=0.096$ \\
\hline & Meniscal extrusion & $\mathrm{R}^{2} 11.2 \%, p=0.002$ & $\mathrm{R}^{2} 6.2 \%, p=0.017$ & NA & NA \\
\hline & Meniscal tears & $\mathrm{R}^{2} 0.02 \%, p=0.91$ & $\mathrm{R}^{2} 0.4 \%, p=0.53$ & $\mathrm{R}^{2} 1.38 \%, p=0.28$ & $\mathrm{R}^{2} 1.9 \%, p=0.21$ \\
\hline & $\begin{array}{l}\text { Tibial + femoral } \\
\text { cartilage defects }\end{array}$ & $\mathrm{R}^{2} 4.5 \%, p=0.051$ & $\mathrm{R}^{2} 2.3 \%, p=0.14$ & $\mathrm{R}^{2} 0.49 \%, p=0.53$ & $\mathrm{R}^{2} 1.1 \%, p=0.33$ \\
\hline & & & Total R² $16.7 \%$ & & Total R $5.6 \%$ \\
\hline
\end{tabular}

Results obtained using linear regression

${ }^{a}$ Adjusted for change in cartilage volume, change in meniscal extrusion, change in meniscal tears or change in cartilage defects where applicable

NA indicates not applicable (incidence of meniscal extrusion very low)

Bold text indicates statistically significant result $(p \leq 0.05)$

$R^{2}$ for univariable models is the proportion of variance explained provided $\left(R^{2}\right)$ for the linear regression

$R^{2}$ for multivariable models is the proportion of variance explained for individual components of the multivariable model (semipartial $R^{2}$ )

Total $R^{2}$ is the proportion of variance explained for the entire multivariable model

effect did not reach statistical significance in any groups or sites, and the magnitude of the effect was less than meniscal extrusion. Additivivity of factors is limited as increases in $\mathrm{R}^{2}$ were modest.

Repeating the analysis using another assessment method for cartilage (average thickness) yielded even weaker associations.

In this study, more than $85 \%$ of change in JSW over time was unexplained by cartilage volume loss, change in meniscal tears, meniscal extrusions or change in cartilage defects. Additionally, the fact that we had to exclude $1 / 3$ of radiographs ( $>2 \mathrm{~mm}$ difference in alignment between films) despite a standardised protocol also suggests the weakness of change in JSW as a measurement over time.

In addition to the factors we measured, the large proportion of unexplained variance could be partially attributed to measurement error due to artefacts in positioning study participants for $\mathrm{x}$-ray. We minimised this through our analysis design, by limiting $\mathrm{x}$-ray data to those whose tibial inter rim distance was $\leq 2 \mathrm{~mm}$ between phase 1 and phase 3 films [37]. Reading of the films themselves is unlikely to add much measurement error, as reproducibility was excellent. Further adjustment for BMI suggests that $\mathrm{BMI}$ is not the major source of unexplained variance. Overall, measurement error due to technical issues remains the most likely explanation given that a substantial proportion of subjects actually increased their JSW over 5 years which is unlikely to be physiologic (unless there is greater cartilage loss in the other compartment). The current FDA and EMEA guidelines accept slowing, cessation or reversal of JSN using conventional radiographs as a structural endpoint for pharmaceutical trials of OA therapies, particularly when accompanied by symptom improvement [3]. However, this study raises the question of whether $\mathrm{x}$-ray measures of JSW should remain the gold standard outcome measure in clinical trials, or 
Table 3 Association between change in cartilage thickness, and new or worsening meniscal extrusions, meniscal tears, and cartilage defects over 2.4 years; and change in joint space width over 5 years $(n=65)$

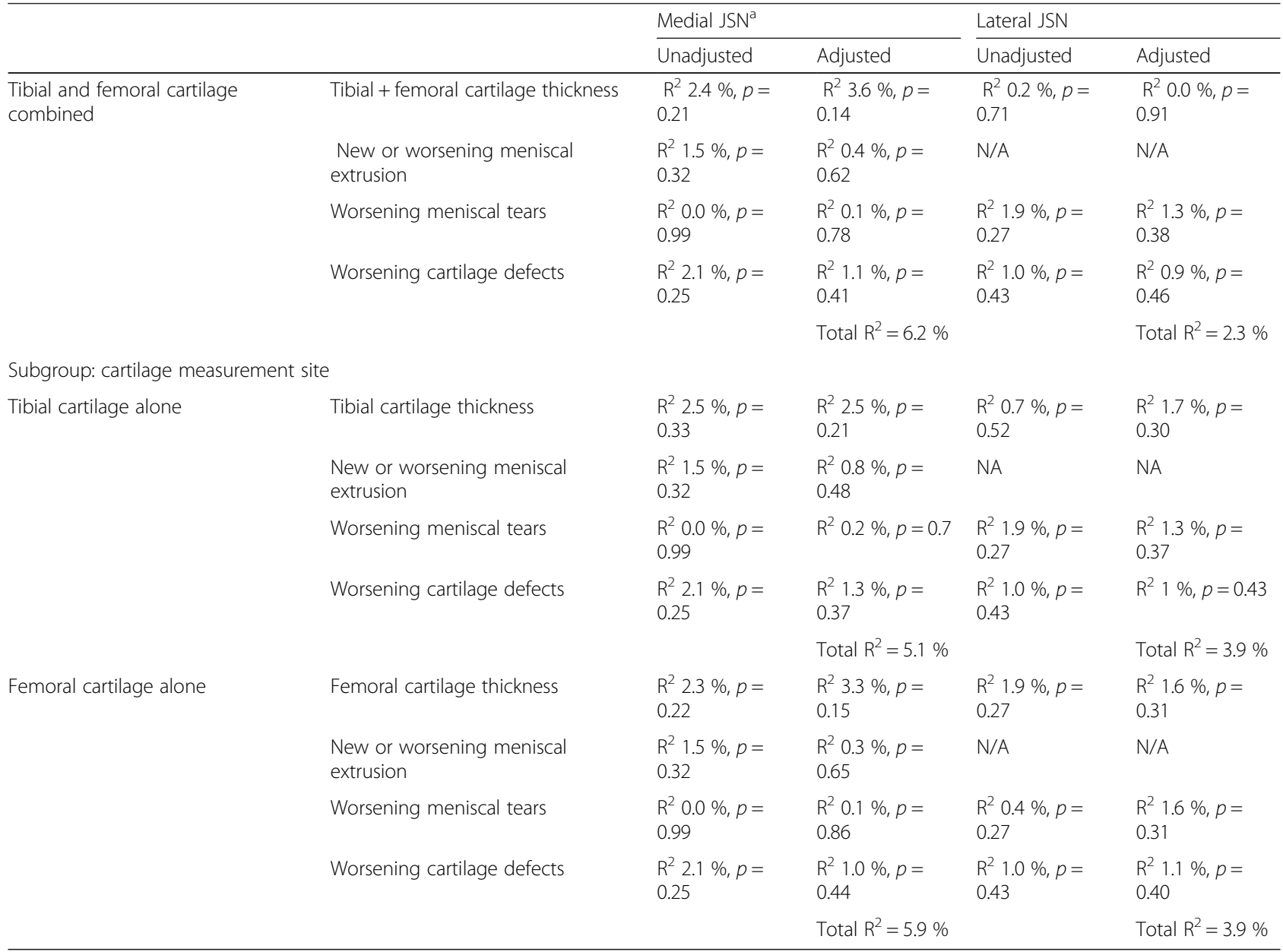

Results obtained using linear regression

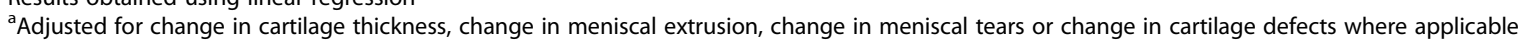
NA indicates not applicable (incidence of meniscal extrusion very low)

Bold text indicates statistically significant result $(p \leq 0.05)$ (No associations were statistically significant)

$R^{2}$ for univariable models is the proportion of variance explained provided $\left(R^{2}\right)$ for the linear regression

$R^{2}$ for multivariable models is the proportion of variance explained for individual components of the multivariable model (semipartial $R^{2}$ )

Total $\mathrm{R}^{2}$ is the proportion of variance explained for the entire multivariable model

whether cartilage loss using MRI should be adopted, as is being proposed by others [38, 39].

There have been a number of longitudinal studies comparing cartilage volume loss and JSW [17, 20-23, 32, 40, 41]. Most focus on the medial compartment of the tibiofemoral joint in populations with OA $[20,21,23,40]$. A cross-sectional association between meniscal extrusion or positioning and JSW has previously been described $[4,7,22,42]$, with one study demonstrating a strong association between medial meniscal subluxation and JSW $(r=0.56)$ [43], and another showing that meniscal position and change in cartilage score both contributed to JSW over 30 months [7]. However, our study is the first to our knowledge that has investigated associations over 5 years, and has used continuous measures of both cartilage volume and JSW.
There are a number of limitations in this study. The gold standard for X-ray protocols have changed since our study began in 2002, based on evidence suggesting that newer methods may be more sensitive to change in JSW [44]. However, the method we used is a sensitive measure of joint space loss over time [45], and studies have shown no advantage of one flexion $\mathrm{x}$-ray protocol over another $[46,47]$ although there are no head to head studies [3]. Additionally, the rates of cartilage loss are lower than might be expected in a population of study participants who all had knee OA. However, sensitivity in our community-based sample [18] is comparable [48], or better [17] than SRM's from other samples. Additionally, we conducted a sensitivity analysis, limiting the data to those with radiographic OA; while the magnitude of the associations increased, the conclusions 
did not change. Another potential criticism is the method used to evaluate meniscal extrusion. Extrusion was examined on sagittal MRI views, and using the same sequence as cartilage so as to limit the time the participant spent in the MRI scanner. Coronal views may be more sensitive [7], and T2 sequences are better for visualising menisci [4]. Therefore, it can be hypothesised that these analyses may underestimate of the contribution of meniscal extrusion to change in JSW. However, this has never been proven by a head to head study. Additionally, the follow up period for Xrays (5 years) and MRI (2.4 years) was not the same. However, as radiograph-assessed measures of $\mathrm{OA}$ progression are less sensitive and require longer periods of observation, this allows additional time for changes that are visible on MR but not on radiographs to become visible, and we do not consider that this affects the conclusions of these analyses. Furthermore, in this study we were only able to examine the sub group of 153 participants in the TASOAC cohort that had full $x$-ray and MRI data and were adequately aligned. However, because there were no significant differences in baseline factors including demographic factors, anthropometry or imaging abnormalities between the participants included in this study and the whole cohort, this suggests that the validity has not been compromised.

\section{Conclusions}

Change in cartilage volume over 2.4 years only weakly predicted change in JSW over 5 years in participants from a community cohort. ME contributed similarly to cartilage volume change to change in JSW in the medial compartment in all study participants, but a greater amount in those with radiographic OA, while changes in meniscal tears and cartilage defects made minimal contributions to proportion of variance explained. Since MRI examines cartilage directly while radiographs examine it indirectly, these results cast doubt on the validity of using JSW as a proxy measure of loss of cartilage volume. This suggests that it is time to re-evaluate this as the choice of primary outcome measure for clinical trials of disease modifying drugs in OA.

\section{Competing interests}

JMP has received consultancy funding from ArthroLab Inc, Abbvie, Bioibérica, Merck \& Co, Servier and TRB Chemedica; expert testimony for Bioibérica and TRB Chemedica; and payment for lectures from Bioibérica, TRB Chemedica and Servier, but these are outside of the submitted work.

JPP has received consultancy funding from ArthroLab Inc, AbbVie, Bioibérica, Elanco, Endocyte, Endocyte, Merck \& Co, Prizer, Sercier, TRB Chemedica; expert testimony for Bioibérica, TRB Chemedica; and payment for lectures for Bioibérica, Servier, TRB Chemedica, but these are outside of the submitted work. FA is an employee of ArthroLab Inc. JMP and JPP own stock or stock options in ArthroLab Inc.

GJ has received grants and personal fees for numerous pharmaceutical companies, but these are outside of the submitted work.

$J H, L L L$, and CD have no competing interests to declare.

\section{Authors' contributions}

GJ designed the original TASOAC study. GJ and FC obtained funding for the study. FC also participated in data collection. CD measured tibial cartilage morphology. JMP JPP and FA provided intellectual property regarding femoral cartilage volume and provided this data. Analyses were designed by GJ and LLL and conducted by JH and LLL. All authors drafted the article and critically revised it for important intellectual content, and approved the final version of the article.

\section{Acknowledgements}

We thank the study participants, who made this study possible, and Catrina Boon and Pip Boon for their role in collecting the data. The authors also thank Rob Warren, André Pelletier and Josée Thériault for the MRI reading, Dr Oliver Stannus for his expertise in assessment of cartilage thickness, and Drs. Marie-Josée Berthiaume and Thomas Moser, specialists in musculoskeletal radiology, for their expert advice on the reading of MR images.

Funding sources: National Health and Medical Research Council of Australia (302204), the Tasmanian Community Fund (D0015018), the Arthritis Foundation of Australia (MRI06161), and the University of Tasmania Grant-Institutional Research Scheme (D0015019).

Dr Laslett is supported by an Arthritis Foundation Australia - Australian Rheumatology Association Heald Fellowship and a National Health and Medical Research Council Early Career Fellowship.

Professor Ding is supported by an Australian Research Council Future Fellowship.

Prof Jones is supported by a National Health and Medical Research Council Practitioner Fellowship.

\section{Author details}

${ }^{1}$ Menzies Institute for Medical Research, University of Tasmania, Private Bag 23, Hobart, Tasmania 7000, Australia. ${ }^{2}$ Osteoarthritis Research Unit, University of Montreal Hospital Research Centre (CRCHUM), Montreal, Quebec, Canada. ${ }^{3}$ Imaging Research and Development, ArthroLab Inc., Montreal, Quebec, Canada. ${ }^{4}$ Department of Epidemiology and Preventive Medicine, School of Public Health and Preventative Medicine, Monash University, Alfred Hospital, Prahran 3181 Melbourne, Australia. ${ }^{5}$ Arthritis Research Institute, 1st Affiliated Hospital, Anhui Medical University, Hefei, Anhui, China.

Received: 20 May 2015 Accepted: 6 January 2016

Published online: 14 January 2016

\section{References}

1. Murray CJ, Vos T, Lozano R, Naghavi M, Flaxman AD, Michaud C, et al. Disability-adjusted life years (DALYs) for 291 diseases and injuries in 21 regions, 1990-2010: a systematic analysis for the Global Burden of Disease Study 2010. Lancet. 2012;380:2197-223.

2. Altman R, Asch E, Bloch D, Bole G, Borenstein D, Brandt $K$, et al. Development of criteria for the classification and reporting of osteoarthritis. Classification of osteoarthritis of the knee. Diagnostic and therapeutic criteria committee of the American Rheumatism Association. Arthritis Rheum. 1986:29:1039-49.

3. Conaghan PG, Hunter DJ, Maillefert JF, Reichmann WM, Losina E. Summary and recommendations of the OARSI FDA osteoarthritis assessment of structural change working group. Osteoarthritis Cartilage. 2011;19:606-10.

4. Berthiaume MJ, Raynauld JP, Martel-Pelletier J, Labonte F, Beaudoin G, Bloch DA, et al. Meniscal tear and extrusion are strongly associated with progression of symptomatic knee osteoarthritis as assessed by quantitative magnetic resonance imaging. Ann Rheum Dis, 2005:64:556-63.

5. Adams JG, McAlindon T, Dimasi M, Carey J, Eustace S. Contribution of meniscal extrusion and cartilage loss to joint space narrowing in osteoarthritis. Clin Radiol. 1999;54:502-6.

6. Hunter DJ, Buck R, Vignon E, Eckstein F, Brandt K, Mazzuca SA, et al. Relation of regional articular cartilage morphometry and meniscal position by MRI to joint space width in knee radiographs. Osteoarthritis Cartilage. 2009:17:1170-6.

7. Hunter DJ, Zhang YQ, Tu X, Lavalley M, Niu JB, Amin S, et al. Change in joint space width: hyaline articular cartilage loss or alteration in meniscus? Arthritis Rheum. 2006;54:2488-95.

8. Ding C, Garnero P, Cicuttini F, Scott F, Cooley H, Jones G. Knee cartilage defects: association with early radiographic osteoarthritis, decreased 
cartilage volume, increased joint surface area and type II collagen breakdown. Osteoarthritis Cartilage. 2005;13:198-205.

9. Mazzuca SA, Brandt KD, Schauwecker DS, Katz BP, Meyer JM, Lane KA, et al. Severity of joint pain and Kellgren-Lawrence grade at baseline are better predictors of joint space narrowing than bone scintigraphy in obese women with knee osteoarthritis. J Rheumatol. 2005;32:1540-6.

10. Saunders J, Ding C, Cicuttini F, Jones G. Radiographic osteoarthritis and pain are independent predictors of knee cartilage loss: a prospective study. Intern Med J. 2011:42:274-80.

11. Bruyere O, Pavelka K, Rovati LC, Deroisy R, Olejarova M, Gatterova J, et al. Glucosamine sulfate reduces osteoarthritis progression in postmenopausal women with knee osteoarthritis: evidence from two 3-year studies. Menopause. 2004;11:138-43.

12. Brandt KD, Mazzuca SA, Katz BP, Lane KA, Buckwalter KA, Yocum DE, et al. Effects of doxycycline on progression of osteoarthritis: results of a randomized, placebo-controlled, double-blind trial. Arthritis Rheum. 2005;52: 2015-25.

13. Michel BA, Stucki G, Frey D, De Vathaire F, Vignon E, Bruehlmann P, et al. Chondroitins 4 and 6 sulfate in osteoarthritis of the knee: a randomized, controlled trial. Arthritis Rheum. 2005;52:779-86.

14. Reginster JY, Deroisy R, Rovati LC, Lee RL, Lejeune E, Bruyere O, et al. Longterm effects of glucosamine sulphate on osteoarthritis progression: a randomised, placebo-controlled clinical trial. Lancet. 2001;357:251-6.

15. Bingham 3rd CO, Buckland-Wright JC, Garnero P, Cohen SB, Dougados M, Adami S, et al. Risedronate decreases biochemical markers of cartilage degradation but does not decrease symptoms or slow radiographic progression in patients with medial compartment osteoarthritis of the knee: results of the two-year multinational knee osteoarthritis structural arthritis study. Arthritis Rheum. 2006;54:3494-507.

16. Hunter DJ, Zhang W, Conaghan PG, Hirko K, Menashe L, Reichmann WM, et al. Responsiveness and reliability of MRI in knee osteoarthritis: a metaanalysis of published evidence. Osteoarthritis Cartilage. 2011;19:589-605.

17. Duryea J, Neumann G, Niu J, Totterman S, Tamez J, Dabrowski C, et al. Comparison of radiographic joint space width with magnetic resonance imaging cartilage morphometry: analysis of longitudinal data from the Osteoarthritis Initiative. Arthritis Care Res (Hoboken). 2010;62:932-7.

18. Aitken D, Ding C, Pelletier JP, Martel-Pelletier J, Cicuttini F, Jones G. Responsiveness of magnetic resonance imaging-derived measures over 2.7 years. J Rheumatol. 2014;41:2060-7.

19. Reichmann WM, Maillefert JF, Hunter DJ, Katz JN, Conaghan PG, Losina E. Responsiveness to change and reliability of measurement of radiographic joint space width in osteoarthritis of the knee: a systematic review. Osteoarthritis Cartilage. 2011;19:550-6.

20. Bruyere O, Genant $H$, Kothari M, Zaim S, White D, Peterfy C, et al. Longitudinal study of magnetic resonance imaging and standard X-rays to assess disease progression in osteoarthritis. Osteoarthritis Cartilage. 2007;15: 98-103.

21. Cicuttini F, Hankin J, Jones G, Wluka A. Comparison of conventional standing knee radiographs and magnetic resonance imaging in assessing progression of tibiofemoral joint osteoarthritis. Osteoarthritis Cartilage. 2005;13:722-7.

22. Raynauld JP, Martel-Pelletier J, Berthiaume MJ, Beaudoin G, Choquette D, Haraoui $B$, et al. Long term evaluation of disease progression through the quantitative magnetic resonance imaging of symptomatic knee osteoarthritis patients: correlation with clinical symptoms and radiographic changes. Arthritis Res Ther. 2006;8:R21.

23. Hunter DJ, Zhang YQ, Niu JB, Tu X, Amin S, Clancy M, et al. The association of meniscal pathologic changes with cartilage loss in symptomatic knee osteoarthritis. Arthritis Rheum. 2006;54:795-801.

24. Lequesne $M$. Quantitiative measurements of joint space during progression of osteoarthritis: chondometry. In: Keuttner K \& Goldberg V, editors. Osteoarthritic Disorders. Rosemont: American Academy of Orthopaedic Surgeons; 1995. p. 427-44.

25. Buckland-Wright C. Protocols for precise radio-anatomical positioning of the tibiofemoral and patellofemoral compartments of the knee. Osteoarthritis Cartilage. 1995;3:71-80.

26. Dupuis DE, Beynnon BD, Richard MJ, Novotny JE, Skelly JM, Cooper SM. Precision and accuracy of joint space width measurements of the medial compartment of the knee using standardized MTP semi-flexed radiographs. Osteoarthritis Cartilage. 2003;11:716-24.
27. Buckland-Wright JC, Ward RJ, Peterfy C, Mojcik CF, Leff RL. Reproducibility of the semiflexed (metatarsophalangeal) radiographic knee position and automated measurements of medial tibiofemoral joint space width in a multicenter clinical trial of knee osteoarthritis. J Rheumatol. 2004;31:1588-97.

28. Altman RD, Hochberg M, Murphy Jr WA, Wolfe F, Lequesne M. Atlas of individual radiographic features in osteoarthritis. Osteoarthritis Cartilage. 1995:3:3-70.

29. Foley SJ, Lord SR, Srikanth V, Cooley H, Jones G. Falls risk is associated with pain and dysfunction but not radiographic osteoarthritis in older adults: Tasmanian older adult cohort study. Osteoarthritis Cartilage. 2006;14:533-9.

30. Jones $G$, Glisson M, Hynes $K$, Cicuttini F. Sex and site differences in cartilage development: a possible explanation for variations in knee osteoarthritis in later life. Arthritis Rheum. 2000;43:2543-9.

31. Ding C, Cicuttini F, Blizzard L, Jones G. Smoking interacts with family history with regard to change in knee cartilage volume and cartilage defect development. Arthritis Rheum. 2007;56:1521-8.

32. Raynauld JP, Martel-Pelletier J, Berthiaume MJ, Labonte F, Beaudoin G, de Guise JA, et al. Quantitative magnetic resonance imaging evaluation of knee osteoarthritis progression over two years and correlation with clinical symptoms and radiologic changes. Arthritis Rheum. 2004:50:476-87.

33. Raynauld JP, Kauffmann C, Beaudoin G, Berthiaume MJ, de Guise JA, Bloch $D A$, et al. Reliability of a quantification imaging system using magnetic resonance images to measure cartilage thickness and volume in human normal and osteoarthritic knees. Osteoarthritis Cartilage. 2003;11:351-60.

34. Stannus OP, Jiang D, Cicuttini F, Cao Y, Ding C. Cartilage signal intensity on T1-weighted MRI: association with risk factors and measures of knee osteoarthritis. Clin Rheumatol. 2014;33:359-68.

35. Cicuttini F, Forbes A, Morris K, Darling S, Bailey M, Stuckey S. Gender differences in knee cartilage volume as measured by magnetic resonance imaging. Osteoarthritis Cartilage. 1999;7:265-71.

36. Cicuttini FM, Jones G, Forbes A, Wluka AE. Rate of cartilage loss at two years predicts subsequent total knee arthroplasty: a prospective study. Ann Rheum Dis. 2004;63:1124-7.

37. Vignon E, Brandt KD, Mercier C, Hochberg M, Hunter D, Mazzuca S, et al. Alignment of the medial tibial plateau affects the rate of joint space narrowing in the osteoarthritic knee. Osteoarthritis Cartilage. 2010;18:1436-40.

38. Eckstein F, Guermazi A, Gold G, Duryea J, Hellio Le Graverand MP, Wirth W, et al. Imaging of cartilage and bone: promises and pitfalls in clinical trials of osteoarthritis. Osteoarthritis Cartilage. 2014;22:1516-32.

39. Guermazi A, Roemer FW, Burstein D, Hayashi D. Why radiography should no longer be considered a surrogate outcome measure for longitudinal assessment of cartilage in knee osteoarthritis. Arthritis Res Ther. 2011;13:247.

40. Cromer MS, Bourne RM, Fransen M, Fulton R, Wang SC. Responsiveness of quantitative cartilage measures over one year in knee osteoarthritis: comparison of radiography and MRI assessments. J Magn Reson Imaging. 2014;39:103-9.

41. Le Graverand MP, Buck RJ, Wyman BT, Vignon E, Mazzuca SA, Brandt KD, et al. Change in regional cartilage morphology and joint space width in osteoarthritis participants versus healthy controls: a multicentre study using 3.0 Tesla MRl and Lyon-Schuss radiography. Ann Rheum Dis. 2010;69:155-62.

42. Bloecker K, Wirth W, Hunter DJ, Duryea J, Guermazi A, Kwoh CK, et al. Contribution of regional $3 \mathrm{D}$ meniscus and cartilage morphometry by MRI to joint space width in fixed flexion knee radiography-a between-knee comparison in subjects with unilateral joint space narrowing. Eur J Radiol. 2013;82:e832-9.

43. Gale DR, Chaisson CE, Totterman SM, Schwartz RK, Gale ME, Felson D. Meniscal subluxation: association with osteoarthritis and joint space narrowing. Osteoarthritis Cartilage. 1999;7:526-32.

44. Le Graverand MP, Mazzuca S, Lassere M, Guermazi A, Pickering E, Brandt K, et al. Assessment of the radioanatomic positioning of the osteoarthritic knee in serial radiographs: comparison of three acquisition techniques. Osteoarthritis Cartilage. 2006;14:A37-43.

45. Nevitt MC, Peterfy C, Guermazi A, Felson DT, Duryea J, Woodworth T, et al. Longitudinal performance evaluation and validation of fixed-flexion radiography of the knee for detection of joint space loss. Arthritis Rheum. 2007;56:1512-20.

46. Mazzuca SA, Brandt KD, Buckwalter KA. Detection of radiographic joint space narrowing in subjects with knee osteoarthritis: longitudinal comparison of the metatarsophalangeal and semiflexed anteroposterior views. Arthritis Rheum. 2003;48:385-90. 
47. Piperno M, Hellio Le Graverand MP, Conrozier T, Bochu M, Mathieu P, Vignon E. Quantitative evaluation of joint space width in femorotibial osteoarthritis: comparison of three radiographic views. Osteoarthritis Cartilage. 1998;6:252-9.

48. Hunter DJ, Zhang W, Conaghan PG, Hirko K, Menashe L, Li L, et al. Systematic review of the concurrent and predictive validity of MRI biomarkers in OA. Osteoarthritis Cartilage. 2011;19:557-88.

Submit your next manuscript to BioMed Central and we will help you at every step:

- We accept pre-submission inquiries

- Our selector tool helps you to find the most relevant journal

- We provide round the clock customer support

- Convenient online submission

- Thorough peer review

- Inclusion in PubMed and all major indexing services

- Maximum visibility for your research

Submit your manuscript at www.biomedcentral.com/submit 